\title{
PROPOSING A CONCEPTUAL FRAMEWORK OF TRANSLATION MODEL (SHORT COMMUNICATION)
}

\section{SANJAY KUMAR JHA}

Amity University Haryana, India

\begin{abstract}
ABSTACT
The objective of this short communication is to propose a theoretical translating model (with both probabilistic and deterministic approach) to achieve the utmost degree of translatability in both human and machine translation. Since the secret of utmost degree of translatability (in terms of naturalness and correctness) lies in the overall linguistic competence of the expert person or expert system, the model primarily concentrates on various issues (faced in human and machine translation of English-Hindi) from linguistic perspectives only.
\end{abstract}

KEYWORDS: Translating Model, Concentrates \& Linguistic

Received: Apr 30, 2018; Accepted: May 19, 2018; Published: May 31, 2018; Paper Id.: IJELJUN201812

\section{INTRODUCTION}

No matter be it human or machine translation, (MT), it is imperative to stress here that one should not muddle up linguistic competence with the competence in a computer programming language. Competence in programming language for MT is just a media of processing linguistic competence in a particular form to make machine understand linguistic instructions for producing the same result what human translator does. What is common and required for both human and machine is an adequate linguistic competence or knowledge base. How good one (human or machine) can translate depends on how good knowledge base one or it has. Therefore, no matter be it human or machine translation, it is the degree of our linguistic competence or knowledge base, which affects and matters in the degree of translatability. The choice of hardware and programming language(s), the definition of a formalism and a user language, the incorporation of world language and statistical data, the maintenance of large dictionaries and terminology collections are all interesting and difficult problems in their own right, but whatever choices or solutions one proposes in these areas, they will have to be integrated in a system which-by necessity- deals with linguistic data (Frank, 1993: vi). Therefore, issues like efficiency in a programming language and several others related to artificial intelligence are of course a matter of debate in their own right, but they are not what the intended model (as a part of the present research) is going to deal with.

Prior to the presentation of the theoretical translating model, it seems plausible to show the status of the present research under (the scope of the translation studies: Holmes (1972) and (the connotations of the term translation by Roger Bell, 1995:13) as follows: 


\section{Scope of Translation}

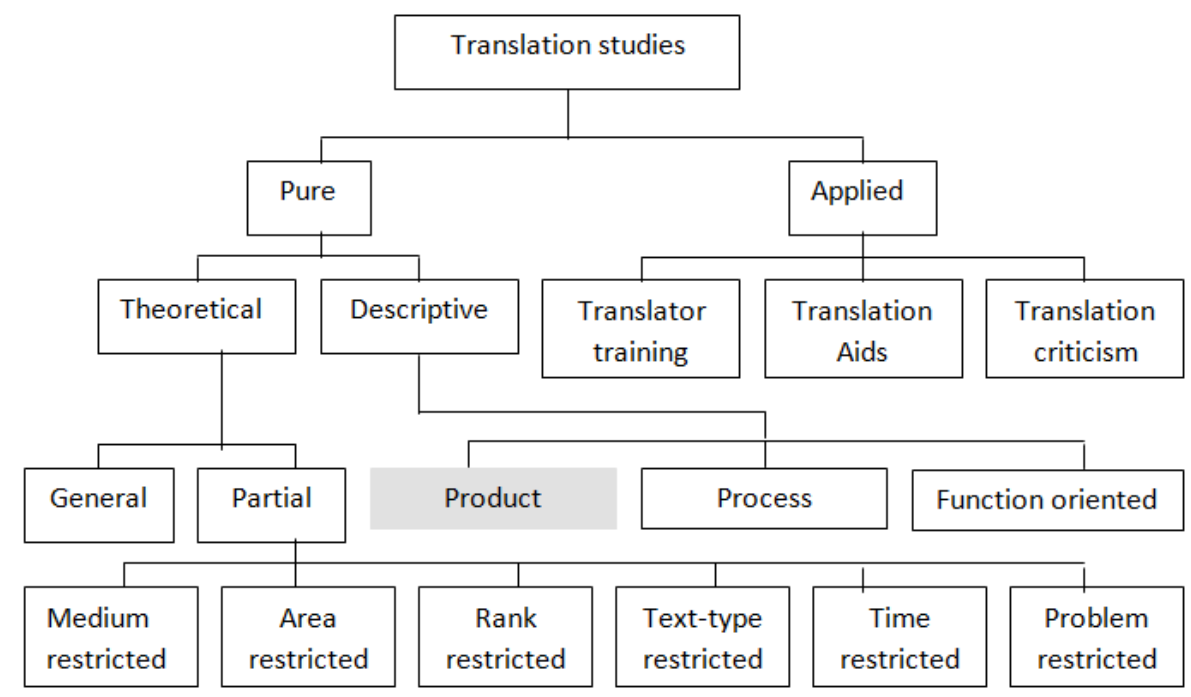

Figure 1: Scope of Translation

\section{Connotations of Translation}

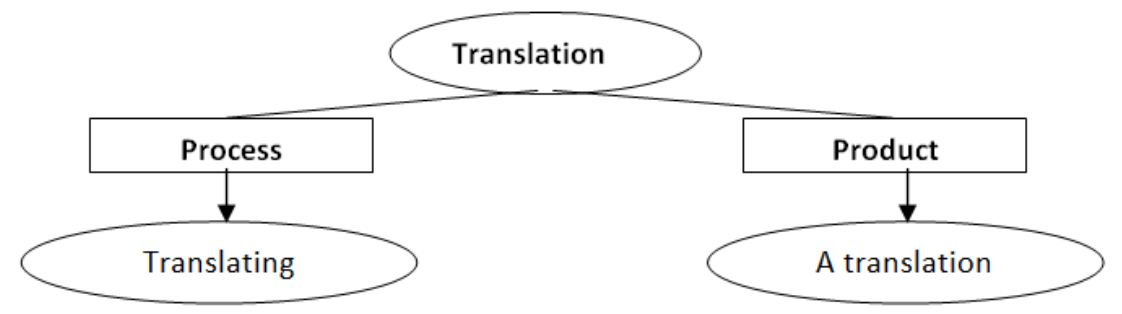

Figure 2: Connotations of Translation

The figure (2) says that 'translation' is an abstract concept, which encompasses both the process (i.e. translating) and the product of that process (i.e. a translation). Viewing the wide spectrum of the translation studies as shown in the figure (1) and the distinctive connotations of the term translation as shown in the figure (2), the present research is primarily product-oriented and secondarily process-oriented. It is primarily product-oriented in a sense that it analyses and evaluates the existing translations produced by three human translators and three machine translation systems as part of the case studies. Secondarily, it is process-oriented in a sense that it suggests different probabilistic and deterministic strategies for human and machine in the form of proposing a common theoretical translating model. 


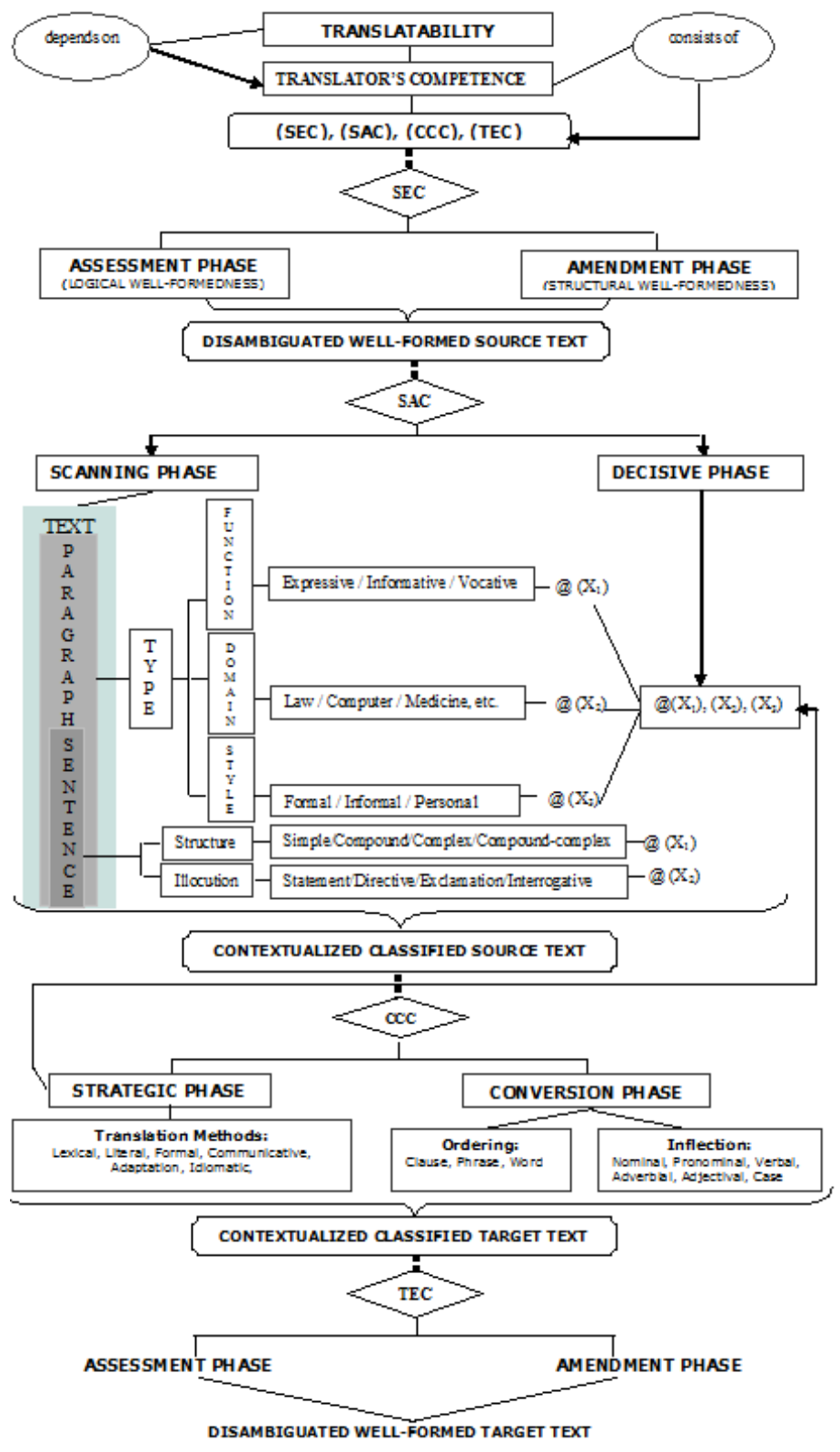

Figure 3: Common Theoretical Translating Model

The aim of this study (in the form of short communication) is to discuss the functioning of a translation model postulated by the researcher. Giving an inverted pyramid description of the above model, 'translatability' is on top which implies that translatability depends on having four types of competence or knowledge base. They are (1) source evaluating competence, (2) source analyzing competence, (3) contrastive conversion competence, (4) target evaluating competence. Lack of any of these competence in a translator or a machine translation system would mean the person or the system cannot execute the process of translating.

The proposed model is both Probabilistic and deterministic - probabilistic in that the model tries to be adequate and realistic in terms of incorporating supra linguistic features of Textuality and dispositionality; deterministic in that it determines prescriptive (what to do) and proscriptive (what not to do) instructions with respect to syntactic ordering, morphological inflections, etc. of any pair of languages. 


\section{Source Evaluating Competence (SEC)}

Source evaluating competence is the first competence to have for a translator which is used in two phases successively. The first phase is assessment and the second is amendment. In assessment phase, a translator assesses logical well-formedness of an SL text from supra linguistic perspective with special reference to Textuality comprising coherence, cohesion, intentionality, acceptability, informativity, situationality, intertextuality, Recomposition, and Disambiguation of anaphoric, attachment, coordinating, homonymous, polysemous, metonymical, metaphorical, structural, dangling, pragmatic type. In amendment phase, a translator ensures structural wellformedness from linguistic perspective with respect to major fault prone areas, e.g. concord, parallelism, modifiers, fragments, run-on, and punctuation of a source text. The outcome of the SEC results into a disambiguated well-formed source text.

\section{Source Analyzing Competence (SAC)}

SAC is the second step of translation in the proposed model which assesses the outcome of SEC, i.e. disambiguated well-formed source text under two phases: scanning and decisive.

Scanning phase has two levels of analysis. The first level of analysis involves analyzing two major units of translation: paragraph and sentence. A paragraph type is scanned or investigated in terms of its function, domain, and style. The second level of analysis involves analyzing sentence (the most important unit of translation subsuming clause, phrase, and word) in terms of its structure and illocution as shown in the figure above.

Decisive phase decides or determines the different textual properties scanned under the first and second level of scanning phase. The decisions finalized by decisive phase have been projected in the form of Xs for further processing under strategic phase of $\mathrm{CCC}$ which has been shown by an arrow coming from the strategic phase to the decisive phase. The outcome of the SAC produces contextualized classified source text, which enters the real phase of translation by applying CCC.

\section{Contrastive Conversion Competence (CCC)}

Contrastive conversion competence is the comepetence by which a translator starts performing the task of translation in true sense. Prior to activating CCC, a translator was just an evaluator and assessor of the source text or more in the role of a monolingual translator. A translator comes into the role of translator by inducing his/her contrastive conversion competence. Contrastive conversion competence enables us to convert the contextualized classified SL text into TL with the help of our contrastive knowledge base. CCC is divided under two phases, namely, Strategic and Conversion. Strategic phase can be viewed, at present, as interactive phase in which user decides which method of translation should be applied to a given syntax or discourse in view of their linguistic and supra linguistic features declared under decisive phase. For example, the following conditions say that if the discourse is Expressive in terms of function; and administration in terms of domain then method of translation will be formal.

\section{Conditions for Selecting a Method of Translation}

If

Discourse_function is Expressive and discourse_domain is administrative then 
method of translation will be formal

Translation specifications and text types are very important factors in MT. For example, some domains like weather forecast and medicine have shown good translation. Similarly, in technical translation, the text is usually informative (e.g. how to install or maintain a piece of equipment), and the specifications are usually consistent in terminology and simple in syntax. But the domain like advertising needs a catchy and creative translation not a syntactically similar phrase with lexical substitution. So is the case in legal translation in which it is very important to assign equivalents to larger units than just words and phrases, and adjustments for differences in legal systems can be very important. Similarly, in literary, authoritative, and sacred translation, style is all-important and there is no one set of specifications that all agree on.

In view of the above-mentioned strategy, one may say that a particular text may have different functions (i.e. expressive, informative, and vocative). One may need to go for "hatero functional" translation (Nord, 1997: 54) which is the combination of different translation methods. To apply hatero functional translation, a human translator will require high degree of subtle perception to discern the different communicative functions of the text. Implementing hatero functional translation in machine translation sounds extremely difficult if not impossible for lack of enormous world knowledge.

The second phase of CCC is conversion phase that requires the contrastive knowledge base at different linguistic levels in terms of ordering, inflection, etc.

The outcome of contrastive knowledge base results into contextualized classified target text, which undergoes further processing to ensure the correctness and naturalness of the translated text by inducing one's TEC

\section{Target Evaluating Competence (TEC)}

The rationale behind having TEC lies in the hypothesis that a translation produced after the conversion phase should not be assumed as the final product, rather it should pass through the test of ideal knowledge base for target language. More precisely, the necessity of having TEC was felt after finding some structural flaws in the translated text despite having contrastive knowledge base under CCC. Such a situation arises when human translator or machine translating system lacks the conditions for the formation of certain contrastive rules under conversion phase of contrastive conversion competence. In order to tackle such problems, the need was felt to create an extra knowledge base. Such an extra knowledge base was termed as TEC which is a kind of additional support for CCC in order to recheck and make the translated text structurally more perfect, stylistically more natural, and karmikally more valid.

\section{CONCLUSIONS}

Like SEC, TEC is also processed under assessment and amendment phases. The assessment phase assesses the translated text in terms of ordering, recomposition, ambiguities, and rhetoric. Under amendment phase, the translated text is modified in terms of skewing and grammatical well-formedness by applying the ideal knowledge base for target language. The outcome of TEC results into disambiguated well-formed target text. 


\section{REFERENCES}

1. Bell, R. T. 1995. Translation and Translating. Longman, New York.

2. Frank, E. V. 1993. Linguistic Issues in Machine Translation, Printer Publisher London.

3. Newmark, P. 1998. A textbook of Translation. Prentice Hall, London. 